\title{
Comparing the Effects of Mastery Learning and Lectures on Nurses' Oxygen Therapy Knowledge and Practice: A Quasi-Experimental Study in Southern Iran
}

\section{Sudabeh Ahmadidarrehsima}

Jiroft University of Medical Sciences

\section{Shideh Rafati}

Hormozgan University of Medical Sciences

Mahnaz Jabalbarezi

Jiroft University of Medical Sciences

Foozieh Rafati ( $\square$ foozieh1384@yahoo.com )

Jiroft University of Medical Sciences

\section{Research Article}

Keywords: mastery learning, lectures, oxygen therapy, knowledge, practice, nurses

Posted Date: September 28th, 2021

DOl: https://doi.org/10.21203/rs.3.rs-850577/v1

License: (c) (i) This work is licensed under a Creative Commons Attribution 4.0 International License.

Read Full License 


\section{Abstract}

Background and Aim: Improper administration of oxygen as an expensive drug has dangerous side effects. Nurses are responsible for performing this therapy and even prescribing it in emergencies. This study aimed to determine the effect of two methods of mastery learning and lectures on the oxygen therapy knowledge and practice of nurses in a teaching hospital in southern Iran.

Materials and Methods: This quasi-experimental study was conducted on 50 nurses working in a teaching hospital in southern Iran. The participants were selected using convenience sampling and randomly divided into intervention and control groups. The members of the intervention group attended 4 oxygen therapy master learning sessions, while the members of the control group were trained using the lecture method for 4 sessions. The instruments used for data collection were a demographic information questionnaire and the researcher-made nurses' oxygen therapy knowledge and practice inventories that were completed by the participants in both groups. The data were collected before the intervention and one week and three months after the intervention and analyzed using SPSS software with descriptive and inferential statistics.

Results: The findings indicated that the nurses had poor oxygen therapy knowledge and practice before the intervention. Furthermore, there was no significant difference between the two groups in terms of the mean scores of oxygen therapy knowledge and practice before the intervention. It was also shown that oxygen therapy knowledge and practice were significantly improved one week and three months after the learning mastery intervention in the participants in the intervention group $(P<0.001)$. In contrast, only the oxygen therapy knowledge of the members of the control group showed a significant increase three months after the intervention compared to the pre-intervention stage $(P=0.04)$.

Conclusion: The results of the present study showed that the mastery learning model is an effective model for improving and maintaining the nurses' oxygen therapy knowledge and practice. Since nurses' optimal practice improves the quality of care and increases patient safety, mastery learning can be used as an effective training technique to improve nurses' oxygen therapy practice.

\section{Introduction}

Oxygen therapy is a treatment technique that provides oxygen to the client at a higher concentration than is available in ambient air. Oxygen administration improves cellular metabolism. Thus, oxygen is considered a drug in clinical situations (1). Although oxygen is used in many cardiac, pulmonary, and emergency disorders, it must be prescribed and cared for as a drug, and its dose or concentration must be constantly monitored for its dangerous side effects, like any drug (2). Oxygen poisoning may occur if high concentrations of oxygen (more than 50\%) are administered for a long time (more than 48 hours). Moreover, administration of high concentrations of oxygen in patients with chronic obstructive pulmonary disease causes loss of respiratory stimulation, followed by a progressive increase in arterial carbon dioxide and ultimately death of the patient due to anesthesia, carbon dioxide, and acidosis (3). Another 
side effect of oxygen therapy is mucosal dryness because oxygen is a gas and lacks moisture, so administering oxygen above 4 liters per minute causes dryness of the respiratory tissue and mucous secretions, both of which can lead to obstruction of the airway and increase the risk of infection (4).

Some studies have shown that errors and mistakes during oxygen therapy include decisions about oxygen administration, oxygen control, oxygen modulation, monitoring, and discontinuation of oxygen therapy. There are also problems with oxygen explosion prevention and nebulizer care. Health care professionals and nurses appear to use oxygen therapy experimentally without sufficient knowledge of indication, dosage, side effects, and intoxication. Rood-Deghan et al. (2011) found that $96.2 \%$ of Iranian nurses have poor adherence to oxygen therapy standards (2). The results of another study in Greece showed that $41 \%$ of nurses considered oxygen not as a medicine but as a gas that improves patients' breathing. It was also found that the majority of nurses had no protocol for oxygen therapy in their wards (5). However, a nurse must have sufficient knowledge and skills to carry out treatment and medication orders and examine the patient for the need for treatment and constantly monitor him/her (6). Thus, in the last twenty years, along with the rapid growth of knowledge and technology, scientific discoveries, and the expansion of health systems, the need for nurses with advanced clinical skills, higher knowledge, and awareness in clinical care settings has increased and more attention is paid to clinical settings and learning in the workplace (7). In other words, the complex environment of current health care requires examining the practice of nurses because nurses' knowledge and skills directly affect patient care (8). Hence, clinical education styles play an important role in the development of nursing skills and comprehensive care (9). One of the clinical education styles is mastery learning, which gives a person enough time to achieve learning (10). According to Bloom (1968), mastery learning also leads to practice appraisal against predetermined standards and a clear measure of one's mastery (11). This clinical education style involves a series of steps including setting educational goals, initial assessment, determining the level of mastery, implementation of the training course, formative assessment, correctional education, and summative assessment. Therefore, one of the advantages of this clinical education style is that learners make more efforts due to higher motivation because they know that they can reach the highest mastery level by spending a certain amount of time (12). Furthermore, using this clinical training style guarantees that nurses acquire the minimum necessary competencies to perform the intended procedure (13). Previous studies have demonstrated the effect of mastery learning on the skill of nasogastric (NG) intubation (14), cardiopulmonary resuscitation (15), and central venous catheter placement $(16,17)$

In Iran, oxygen therapy is an essential activity in nursing practice and nurses are responsible for safe medication to the patient (18) and must regularly check the oxygen therapy-based medical instructions so that the patient receives the correct concentration of oxygen. Besides, in Iran, five rules of prescribing drugs, namely the right medicine, the right dose, the right client, the right method, and the right time must be observed for prescribing oxygen to patients (19). Therefore, this study examined the effect of two educational methods, mastery learning, and lectures, on oxygen therapy knowledge and practice of nurses in southern Iran. 


\section{Materials And Methods}

This quasi-experimental study aimed to evaluate the effect of two educational methods, mastery learning, and lectures, on oxygen therapy knowledge and practice of 50 nurses of a teaching hospital in southern Iran. The participants were selected using convenience sampling. To do so, the Continuing Education Center informed the nurses of the training program. Afterward, the enrolled nurses were randomly placed in the two intervention (mastery learning) and control (lecturing) groups. The inclusion criteria were the willingness for voluntary participation in the study and having at least three years of service records as a nurse. The exclusion criterion was the failure to attend the training sessions regularly. The data collection instruments were a demographic information questionnaire, the Nurses' Oxygen Therapy Knowledge Inventory, and the Nurses' Oxygen Therapy Practice Inventory. The demographic information questionnaire contained 7 items that assessed the participants' age, gender, place of residence, marital status, education, clinical records, and the name of the clinic or ward. The Nurses' Oxygen Therapy Knowledge Inventory was a research-made tool that was developed using books and articles in nursing principles and skills $(1,3)$ and contained 20 four-choice items (e.g., What is the right decision for a patient who needs oxygen at your diagnosis but the doctor has not prescribed oxygen for him/her?). The total score on the inventory ranges from 0 to 20 . To calculate each respondent's score, the sum of the scores of the 20 items was calculated and then divided by 20 (the total number of items). Accordingly, the respondent oxygen therapy knowledge was assessed using four quartiles $(25,50$, and 75 percentiles). Accordingly, a score of less than 25 indicated poor oxygen therapy knowledge, a score of 25 to 50 indicated moderate oxygen therapy knowledge, a score of 50 to 75 indicated good oxygen therapy knowledge, and a score of higher than 75 indicated excellent oxygen therapy knowledge. Moreover, the Nurses' Oxygen Therapy Practice Inventory was developed following a review of previous studies in the literature $(2,12,13)$ and contained 20 items (e.g. I check the patient's breathing rate and depth before administering oxygen) that measured the nurses' oxygen therapy practice on a five-point Likert scale (always, often, sometimes, rarely, and never). The total score on the inventory ranges from 0 to 80 . To calculate each respondent's score, the sum of the scores of the 20 items was calculated and then divided by 20 (the total number of items). Then, the respondent oxygen therapy practice was assessed using four quartiles $(25,50$, and 75 percentiles). Hence, a score of less than 25 indicated poor oxygen therapy practice, a score of 25 to 50 indicated moderate oxygen therapy practice, a score of 50 to 75 showed good oxygen therapy practice, and a score of higher than 75 suggested excellent oxygen therapy practice.

The content validity of the nurses' oxygen therapy knowledge and practice inventories was assessed and confirmed by 10 faculty members of the School of Nursing. It should be noted that the content validity of the instruments was assessed both qualitatively and quantitatively by 10 nursing faculty members who had a history of teaching oxygen therapy. Accordingly, the items in the inventories were revised based on the feedback received from the faculty members. The quantitative content validity was assessed using the content validity index (CVI) and content validity ratio (CVR). To calculate the CVR, the items in the inventories were assessed by 10 nursing faculty members and they checked the necessity of each item ( 1 = Necessary, 2 = Useful but not necessary, and $3=$ Not necessary). Thus, the items with CVR less than 0.7 were removed. To measure the $\mathrm{CVI}$, the experts were asked to determine how relevant each item was. 
A score of 0.7 or higher was considered acceptable (20). Moreover, the face validity of the instruments was checked by 10 nurses. To measure the reliability of the instruments, Cronbach's alpha coefficient was calculated which was 0.87 in a pilot study on 20 nurses.

The content of the training program was prepared based on the books on nursing principles and skills. The instructional content covered topics on various issues including the use of oxygen therapy devices (nasogastric (NG) tubes, simple face masks, venturi masks, masks with inhaled oxygen exhalation, etc.), the adjustment of the amount of oxygen according to the doctor's instructions, checking the patient's airway, performing respiratory physiotherapy and incentive spirometry, checking hypoxia symptoms, oxygen therapy complications, determining the patient's oxygen saturation with a pulse oximeter, and oxygen poisoning symptoms.

Before starting the intervention, written consent was obtained from all participants and some information was given to them about the training program. The participants in both groups completed the questionnaires. A 30-minute briefing session was held for the participants in the intervention group to make them familiar with the training protocol. Afterward, the intended nursing skills were instructed to the participants in the intervention group using the mastery learning method through interactive lectures and questions and answers. The instructions were provided by an emergency medicine physician and a nursing professor. At the end of the training sessions (4 training sessions each lasting 1.5 hours), the post-test was administered to the group members. The individuals who scored less than $80 \%$ of the total score were trained individually. Individual training continued until all members obtained at least $80 \%$ of the total grade and gained complete mastery over the topics covered in the training sessions (13). Furthermore, the same educational content was instructed through lectures to the participants in the control group. One week and also three months after the training sessions, the participants in both groups completed the questionnaires and the results were compared.

In this study, continuous variables were described with the mean, standard deviation (SD), median, and interquartile range (IQR), and categorical variables were measured using frequency and percentage. The chi-square test was applied to test the possible associations between the categorical variables. The normality assumption of continuous variables was rejected by the Shapiro-Wilk test ( $P$ value $<0.05$ ). Thus, the Mann-Whitney U test was applied to compare the means of the two groups. Moreover, the nonparametric covariance analysis (ANCOVA) (Quade Method) was used to test the effect of an independent variable (the variable with 2 intervention and control groups) on a continuous dependent variable (nurses' practice or knowledge) by controlling the effect of the nurses' practice or knowledge scores in the baseline time. In addition, the Friedman test and Dunn's pairwise post hoc tests were applied to detect significant differences between the means of the dependent variable at 3-time points. A P $<0.05$ was considered statistically significant and the data were analyzed by SPSS software.

\section{Results}


In this study, the oxygen therapy knowledge and practice of 25 nurses in the intervention group (mastery learning) and 25 nurses in the control group (lecturing) were evaluated before, one week after, and three months after the intervention. There were no significant differences between the two groups in terms of the demographic variables $(P$-value $>0.05)$ as shown in Table 1 : 
Table 1

The participants' demographic characteristics

\begin{tabular}{|c|c|c|c|c|c|c|}
\hline \multirow{2}{*}{\multicolumn{2}{|c|}{ Variable }} & \multicolumn{2}{|c|}{ Intervention Group } & \multicolumn{2}{|c|}{ Control Group } & \multirow[t]{2}{*}{ P-value $^{3}$} \\
\hline & & $\begin{array}{l}\text { Mean } \\
(S D)^{1}\end{array}$ & $\begin{array}{l}\text { Median } \\
(\mathrm{IQR})^{2}\end{array}$ & $\begin{array}{l}\text { Mean } \\
(S D)^{1}\end{array}$ & Median (IQR) ${ }^{2}$ & \\
\hline \multicolumn{2}{|l|}{ Age (year) } & $31.72(5.66)$ & $30.0(3.0)$ & $30.68(4.29)$ & $30.0(4.0)$ & 0.681 \\
\hline \multirow{2}{*}{\multicolumn{2}{|c|}{ Experience (year) }} & $7.20(5.28)$ & $5.0(4.0)$ & $5.84(2.77)$ & $5.0(3.0)$ & 0.371 \\
\hline & & Number & Percent & Number & Percent & P-value ${ }^{4}$ \\
\hline \multirow[t]{2}{*}{ Gender } & Male & 6.0 & 12.0 & 5.0 & 10.0 & \multirow[t]{2}{*}{0.999} \\
\hline & Female & 19.0 & 38.0 & 20.0 & 40.0 & \\
\hline \multirow{2}{*}{$\begin{array}{l}\text { Marital } \\
\text { Status }\end{array}$} & Single & 2.0 & 4.0 & 4.0 & 8.0 & \multirow[t]{2}{*}{0667} \\
\hline & Married & 23.0 & 46.0 & 21.0 & 42.0 & \\
\hline \multirow[t]{2}{*}{ Residence } & Urban & 25.0 & 50.0 & 25.0 & 50.0 & \multirow[t]{2}{*}{-} \\
\hline & Rural & 0.0 & 0.0 & 0.0 & 0.0 & \\
\hline \multirow[t]{2}{*}{ Education } & Bachelor & 24.0 & 48.0 & 23.0 & 46.0 & \multirow[t]{2}{*}{0.999} \\
\hline & Master & 1.0 & 2.0 & 2.0 & 4.0 & \\
\hline \multirow[t]{9}{*}{ Ward } & Emergency & 3.0 & 6.0 & 2.0 & 4.0 & \multirow[t]{9}{*}{0.991} \\
\hline & Covid & 3.0 & 6.0 & 3.0 & 6.0 & \\
\hline & Surgery & 2.0 & 4.0 & 1.0 & 2.0 & \\
\hline & Orthopedic & 4.0 & 8.0 & 6.0 & 12.0 & \\
\hline & ICU & 6.0 & 12.0 & 6.0 & 12.0 & \\
\hline & Pediatrics & 2.0 & 4.0 & 1.0 & 2.0 & \\
\hline & Nerves & 2.0 & 4.0 & 2.0 & 4.0 & \\
\hline & Infectious & 2.0 & 4.0 & 2.0 & 4.0 & \\
\hline & $\mathrm{NICU}$ & 1.0 & 2.0 & 2.0 & 4.0 & \\
\hline
\end{tabular}


Table 2

The relationship between the nurses' knowledge and practice in the intervention and control groups

\section{Variable}

Knowledge score (\%)

Baseline
Intervention group

Control group

P value $^{1}$

\begin{tabular}{|c|c|c|c|c|}
\hline & & & & \\
\hline & Medium & $5.0(10.0 \%)$ & $3.0(6.0 \%)$ & \\
\hline & Good & $10.0(20.0 \%)$ & $12.0(24.0 \%)$ & \\
\hline & Excellent & $6.0(12.0 \%)$ & $6.0(12.0 \%)$ & \\
\hline \multirow[t]{4}{*}{ One week after the intervention } & Low & $0.0(0.0 \%)$ & $12.0(24.0 \%)$ & \multirow[t]{4}{*}{$<0.001$} \\
\hline & Medium & $0.0(0.0 \%)$ & $13.0(26.0 \%)$ & \\
\hline & Good & $11.0(22.0 \%)$ & $0.0(0.0 \%)$ & \\
\hline & Excellent & $14.0(28.0 \%)$ & $0.0(0.0 \%)$ & \\
\hline \multirow[t]{4}{*}{ Three months after the intervention } & Low & $0.0(0.0 \%)$ & $9.0(18.0 \%)$ & \multirow[t]{4}{*}{$<0.001$} \\
\hline & Medium & $0.0(0.0 \%)$ & $15.0(30.0 \%)$ & \\
\hline & Good & $11.0(22.0 \%)$ & $1.0(2.0 \%)$ & \\
\hline & Excellent & $14.0(28.0 \%)$ & $0.0(0.0 \%)$ & \\
\hline \multicolumn{5}{|l|}{ Practice score (\%) } \\
\hline \multirow[t]{4}{*}{ Baseline } & Weak & $5.0(10.0 \%)$ & $4.0(8.0 \%)$ & \multirow[t]{4}{*}{0.925} \\
\hline & Medium & $7.0(14.0 \%)$ & $7.0(14.0 \%)$ & \\
\hline & Good & $6.0(12.0 \%)$ & $8.0(16.0 \%)$ & \\
\hline & Excellent & $7.0(14.0 \%)$ & $6.0(12.0 \%)$ & \\
\hline \multirow[t]{4}{*}{ One week after the intervention } & Weak & $0.0(0.0 \%)$ & $11(22.0 \%)$ & \multirow[t]{4}{*}{$<0.001$} \\
\hline & Medium & $0.0(0.0 \%)$ & $14(28.0 \%)$ & \\
\hline & Good & $8.0(16.0 \%)$ & $0.0(0.0 \%)$ & \\
\hline & Excellent & $17.0(34.0 \%)$ & $0.0(0.0 \%)$ & \\
\hline \multirow[t]{3}{*}{ Three months after the intervention } & Weak & $0.0(0.0 \%)$ & $10.0(20.0 \%)$ & \multirow[t]{3}{*}{$<0.001$} \\
\hline & Medium & $0.0(0.0 \%)$ & $15.0(30.0 \%)$ & \\
\hline & Good & $10.0(20.0 \%)$ & $0.0(0.0 \%)$ & \\
\hline
\end{tabular}




\section{Variable}

\section{Intervention group Control group $\quad P_{\text {value }}{ }^{1}$}

\section{Excellent $\quad 15.0(30.0 \%) \quad 0.0(0.0 \%)$}

${ }^{1}$ The chi-square test

As shown in Table 2, there is no significant association between the learning styles and nurses' oxygen therapy knowledge and practice in the baseline time $(P$-value $=0.877)$ and $(P$-value $=0.925)$, respectively. Unlike the control group, the nurses' knowledge and practice of oxygen therapy in the intervention group improved significantly one week after the intervention and three months later (P-value $<0.001)$.

The results also revealed that the mean scores of nurses' knowledge in the two groups increased significantly compared to the baseline time $(P$-value $<0.05)$. However, unlike the control group, the mean score of nurses' practice in the intervention group increased compared to the baseline time ( $P$-value < 0.001) as displayed in Table 3. Table 4 shows the post hoc analysis of Friedman's Test results. Figure 1 shows error bars for the nurses' practice and knowledge over time.

Table 3

A comparison of the nurses' knowledge and practice in three-time points

\begin{tabular}{|c|c|c|c|c|}
\hline Group & Baseline & $\begin{array}{l}\text { One week after the } \\
\text { intervention }\end{array}$ & $\begin{array}{l}3 \text { months after the } \\
\text { intervention }\end{array}$ & $\begin{array}{l}\text { P- } \\
\text { value }^{1}\end{array}$ \\
\hline \multicolumn{5}{|c|}{ Nurses' Knowledge; Mean (Standard Error) } \\
\hline Intervention & $0.18(0.02)$ & $0.92(0.01)$ & $0.83(0.02)$ & $\begin{array}{l}< \\
0.001^{\mathrm{a}}\end{array}$ \\
\hline Control & $0.18(0.02)$ & $0.21(0.02)$ & $0.26(0.02)$ & $0.042^{\mathrm{a}}$ \\
\hline \multicolumn{5}{|c|}{ Nurses' Practice ${ }^{3}$; Mean (Standard Error) } \\
\hline Intervention & $1.92(0.04)$ & $3.93(0.01)$ & $3.76(0.02)$ & $\begin{array}{l}<.001^{\mathrm{a}} \\
<\end{array}$ \\
\hline Control & $1.94(0.04)$ & $2.07(0.03)$ & $1.99(0.05)$ & 0.075 \\
\hline
\end{tabular}


Table 4

Pairwise Comparisons

\begin{tabular}{|c|c|c|c|c|}
\hline \multirow[t]{2}{*}{ Difference } & \multicolumn{2}{|c|}{ Intervention Group } & \multicolumn{2}{|c|}{ Control Group } \\
\hline & $\begin{array}{l}\text { Test } \\
\text { statistic }^{1}\end{array}$ & $\begin{array}{l}\mathrm{P}- \\
\text { value }\end{array}$ & $\begin{array}{l}\text { Test } \\
\text { statistic }^{1}\end{array}$ & $\begin{array}{l}\mathrm{P}- \\
\text { value }\end{array}$ \\
\hline \multicolumn{5}{|l|}{ Nurses' Knowledge } \\
\hline baseline-one week after learning & -1.80 & $\begin{array}{l}< \\
0.001^{\mathrm{a}}\end{array}$ & -0.28 & 0.322 \\
\hline baseline-3 months after learning & -1.20 & $\begin{array}{l}< \\
0.001^{\mathrm{a}}\end{array}$ & -0.68 & $0.016^{\mathrm{a}}$ \\
\hline 3 months after learning- one week after learning & 0.60 & $0.034^{a}$ & -0.40 & 0.157 \\
\hline \multicolumn{5}{|l|}{ Nurses' Practice } \\
\hline baseline-one week after the intervention & -1.86 & $\begin{array}{l}< \\
0.001^{\mathrm{a}}\end{array}$ & - & - \\
\hline baseline -3 months after the intervention & -1.14 & $\begin{array}{l}< \\
0.001^{\mathrm{a}}\end{array}$ & - & - \\
\hline $\begin{array}{l}3 \text { months after the intervention- one week after the } \\
\text { intervention }\end{array}$ & 0.72 & $0.011^{a}$ & - & - \\
\hline
\end{tabular}

Table 5

The nonparametric ANCOVA to test the effect of learning styles on the nurses' knowledge and practice by controlling the effect of nurses' knowledge or practice in the baseline time

\begin{tabular}{|lll|}
\hline Variable & Test statistic $^{1}$ & P-value \\
\hline The knowledge one week after the intervention & 150.58 & $<0.001$ \\
\hline Knowledge 3 months after the intervention & 148.27 & $<0.001$ \\
\hline Practice one week after the intervention & 152.51 & $<0.001$ \\
\hline Practice 3 months after the intervention & 144.42 & $<0.001$ \\
\hline${ }^{1}$ Quade Method & & \\
\hline
\end{tabular}


The findings displayed in Table 5 showed that by controlling the practice score of the nurses at the baseline time, the mean practice score of the nurses in the intervention group and the control group was different one week and 3 months after the intervention (P-value < 0.001). Moreover, by controlling the knowledge score of nurses at the baseline time, the mean knowledge score of nurses in the intervention group and the control group was different both one week and 3 months after the intervention (P-value < $0.001)$.

\section{Discussion}

The present study evaluated the effect of two educational methods, mastery learning, and lectures, on the oxygen therapy knowledge and practice of nurses in a teaching hospital in southern Iran. The findings suggested that the nurses' oxygen therapy knowledge and practice were poor in both groups before the intervention. Similarly, Hamdy Mostafa et al. (2019) and Aloushan et al. (2019) showed that nurses' oxygen therapy knowledge and skills were not satisfactory $(21,22)$ perhaps due to their inefficiency in updating their knowledge and skills after a long stay in clinical settings and their high workload (23).

The present study also showed that mastery learning was more effective than the lecture method on nurses' oxygen therapy knowledge and practice. In a similar vein, Lengetti et al. (2018) (8) also showed the effectiveness of mastery learning in comparison with the traditional method on retaining nurses' urinary catheter placement skills one month after the training program. Dorri et al. (2018) (13) found that using the mastery learning model can lead to the improvement of practical nursing skills and satisfactory practice of nursing procedures. Furthermore, Barsuk et al. (2009) (24) conducted a descriptiveobservational prospective study to increase the skills of 18 nephrology residents in temporary hemodialysis catheter placement and found that the mastery learning model was effective in increasing their clinical skills. Butter et al. (2010) (25) also conducted a study with a pretest-posttest design and a control group to investigate the effect of mastery learning using a simulator in improving cardiac auscultation skills and confirmed the effectiveness of mastery learning in improving medical students' auscultation skills. Additionally, Benbassat et al. (2007) (26) showed that the mastery learning program is a strategy that can improve nursing students' skills and knowledge in the physical examination course. Tang et al. (2013) (27) implemented mastery learning in a clinical skills training program for primary care nurses and showed that the clinical competence of nurses including knowledge, skills, cooperation, and professional development increased significantly after the application of the mastery learning program, as was confirmed by the data in the present study.

\section{Limitations:}

One of the limitations of this study was related to the individual differences in the participants. Since learning styles and the learning speed differ in individual nurses, it was tried to take into account these differences in this study when implementing the training program. Moreover, the education received by 
nurses from other sources (books, television, etc.) during the training course was out of the control of the researcher. Thus, future studies can explore the effect of the mastery learning model on nurses' practice in other clinical procedures.

\section{Declarations}

\section{Ethics approval and consent to participate:}

This research project was approved by the ethics committee of Jiroft University of Medical Sciences (IR.JMU.REC.1399.030) and All methods were performed in accordance with the relevant guidelines and regulation of the Declaration of Helsinki. Before obtaining informed consent, the necessary explanations about the voluntary participation, anonymity, confidentiality, and safe protection of the data were given to the participants. In addition, the participants were informed that they could leave the study at any time.

\section{Consent for publication:}

Not applicable.

\section{Availability of data and materials:}

The datasets used and/or analyzed during the current study are availablefrom the corresponding authors on reasonable request.

\section{Competing interests:}

The authors reported no conflict of interest.

\section{Funding:}

Self-funded.

\section{Authors' contributions:}

A.S and J. M. performed the measurements, was involved in planning and supervised the work, R. Sh. processed and performed the analysis, drafted the manuscript and designed the tabels. They wrote the manuscript with scientific support and helped supervise the project. R. F. involved in interpreting the results, worked on the manuscript and edited it. All authors discussed the results and commented on the manuscript. All authors read the final copy of manuscript and confirmed it for submission.

\section{Acknowledgments:}

The authors would like to appreciate the Vice-Chancellor for Research and Technology of Jiroft University of Medical Sciences and the sincere and honest cooperation of the nurses of Imam Khomeini Hospital in Jiroft who contributed to conducting this study. 


\section{Authors' information:}

Sudabeh Ahmadidarrehsima ${ }^{1}, \mathrm{RN}, \mathrm{MSc}$

Shideh Rafati ${ }^{2}$, MSc. PhD.

Mahnaz Jabalbarezi ${ }^{3}$, RN.

Foozieh Rafati ${ }^{4}$, MSc. PhD.

1-Email: sudabeh.Ahmadi1122@gmail.com.Tell: 09136124195.

PO Box:7861763730. Address: Shahid Hajghasem Solymani Square, Jiroft University of Medical Sciences, Jiroft, Kerman, Iran.

2 Email: shiderafati@yahoo.com . Tell: 09376651275. PO Box: 7916193145 . Address: Emam Khomeini Boulevard - North Golshahr - Daru Pakhsh Street, Hormozgan Health Institute, Hormozgan University of Medical Sciences, Bandar Abbas, Iran .

3-E.mail: m.jabalbarezi93@gmail.com. Tell: +9809132491246. PO Box:7861763730. Address: Emam Khomeini Educational and Medical Hospital , Jiroft University of Medical Sciences, Jiroft, Kerman, Iran.

4*-Corresponding author: Email: foozieh1384@yahoo.com. Tell: 09133489902.

PO Box:7861763730. Address: Shahid Hajghasem Solymani Square, Jiroft University of Medical Sciences, Jiroft, Kerman, Iran

\section{References}

1. Saeidi M, oxygen delivery. in: Berman A, Synder SH, Frandsen G. Kozier \& Erbs Fundamentals of Nursing: concepts, process, and practice. 10th ed, Tehran, Heidary press 2018; P $389-415$.

2. Rood-Deghan Z, et al. Staff nurses' adherence to oxygen therapy standards. Iran Journal of Nursing 2011; 23(68) : 8-18. [Persian]

3. Deghan Nayeri N, and et al. oxygen delivery. In: Potter P, Particia A. Fundamentals of nursing potter perry. Tehran, Andishe rafi 2014; P 693 - 769.

4. Shaban M, and Latifi M. in Janice L, Carrie H. Bruner \& Sudarth 2018: breathing and gas exchanges. Tehran, Boshra press 2018; P 145 - 168.

5. Brokalaki H, Matziou V, Zyga S, Kapella M, Tsaras K, Brokalaki E, and Myrianthefs P. Omissions and errors during oxygen therapy of hospitalized patients in a large city of Greece. Intensive and Critical Care Nursing 2004; 20(6): 352-357.

6. Gonca Hanedan U, Gamze C, Cobanglo B. Hyperbaric oxygen therapy in the treatment of complications of irradiation in laryngeal cancer. Contemporary Oncology 2018; 22(3): 202. 
7. Parandeh A, and Hage Amini Z. Practice-based learning and its outcomes in nursing students' education. Education \& Ethics in Nursing 2013; 2(3): 1-8. [Persian]

8. Lengettil $\mathrm{E}$, et al. An innovative approach to educating nurses to clinical competence: A randomized controlled trial. Nurse education in practice 2018; 33: 159-163.

9. Karimi Moonaghi $\mathrm{H}$, and et al. Teaching style in clinical nursing education: A qualitative study of Iranian nursing teachers' experiences. Nurse education in practice 2010; 10(1): 8-12. [Persian]

10. Guskey T. R, Anderman E. M. In search of a useful definition of mastery. Educational Leadership 2013; 71(4): 18.

11. Bloom B. S. Learning for Mastery. Evaluation Comment 1968; 1(2): 1-12.

12. Vizeshfar $F$, and Torabizadeh $C$. The effect of teaching based on dominant learning style on nursing students' academic achievement. Nurse education in practice 2018; 28: 103-108.

13. Dorri S, and Hakimi H. The Effect of Mastery learning model for suction and oxygen therapy skills in nursing students. Research in Medical Education 2018; 9(4): 19-10. [Persian]

14. Eppich W. J, Hunt E. A, Duval-Arnould J. M, Siddall V. J, \& Cheng A. Structuring feedback and debriefing to achieve mastery learning goals. Academic Medicine 2015; 90(11): 1501-1508.

15. Roh Y. S, Lim E. J, \& Issenberg S. B. Effects of an integrated simulation-based resuscitation skills training with clinical practicum on mastery learning and self-efficacy in nursing students. Collegian 2016; 23(1): 53-59.

16. Barsuk J. H, Cohen E. R, Williams M. V, Scher J, Feinglass J, McGaghie W. C, \& Wayne D. B. The effect of simulation-based mastery learning on thoracentesis referral patterns. Journal of hospital medicine 2016; 11(11): 792-795.

17. D'amore A, Santhamma J, and Mitchell E KL. Learning styles of first-year undergraduate nursing and midwifery students: A cross-sectional survey utilizing the Kolb Learning Style Inventory. Nurse education today 2012; 32(5): 506-515.

18. Kord Z, and et al. Auditing of oxygen therapy nursing care in premature neonatal in NICU training centers of Ahvaz University of Medical Sciences in 2012. JPEN 2015; 2(1): 9-18. [Persian]

19. Potter P, Perry A. Basic nursing concepts and application. St.Louis: Mosby 2014; P.692.

20. Polit DF, Beck CT. Essentials of nursing research: Appraising evidence for nursing practice: Lippincott Williams \& Wilkins; 2009.

21. Hamdy Mostafa A, and et al. Effect of Educational Program on Nurses' Knowledge and Practice About Oxygen Therapy. Assiut Scientific Nursing Journal 2019; 7(18): 95-104.

22. Aloushan A F, and et al. Assessment of knowledge, attitude and practice regarding oxygen therapy at emergency departments in Riyadh in 2017: A cross-sectional study. World journal of emergency medicine 2019; 10(2): 88.

23. Vargas F, and et al. Physiologic effects of high-flow nasal cannula oxygen in critical care subjects. 2015. 
24. Barsuk J, and et al. Mastery learning of temporary hemodialysis catheter insertion by nephrology fellows using simulation technology and deliberate practice. American Journal of Kidney Diseases 2009; 54(1): 70-76.

25. Butter $\mathrm{J}$, and et al. Simulation-based mastery learning improves cardiac auscultation skills in medical students. Journal of general internal medicine 2010; 25(8): 780-785.

26. Benbassat J, Baumal R. A proposal for teaching basic clinical skills for mastery: the case against vertical integration. Academic Medicine 2007; 82(1): 83-91.

27. Tang W, Dong L. Applying mastery learning in a clinical skills training program for primary care nurses. The Journal of Continuing Education in Nursing 2013; 44(12): 535-541.

\section{Figures}




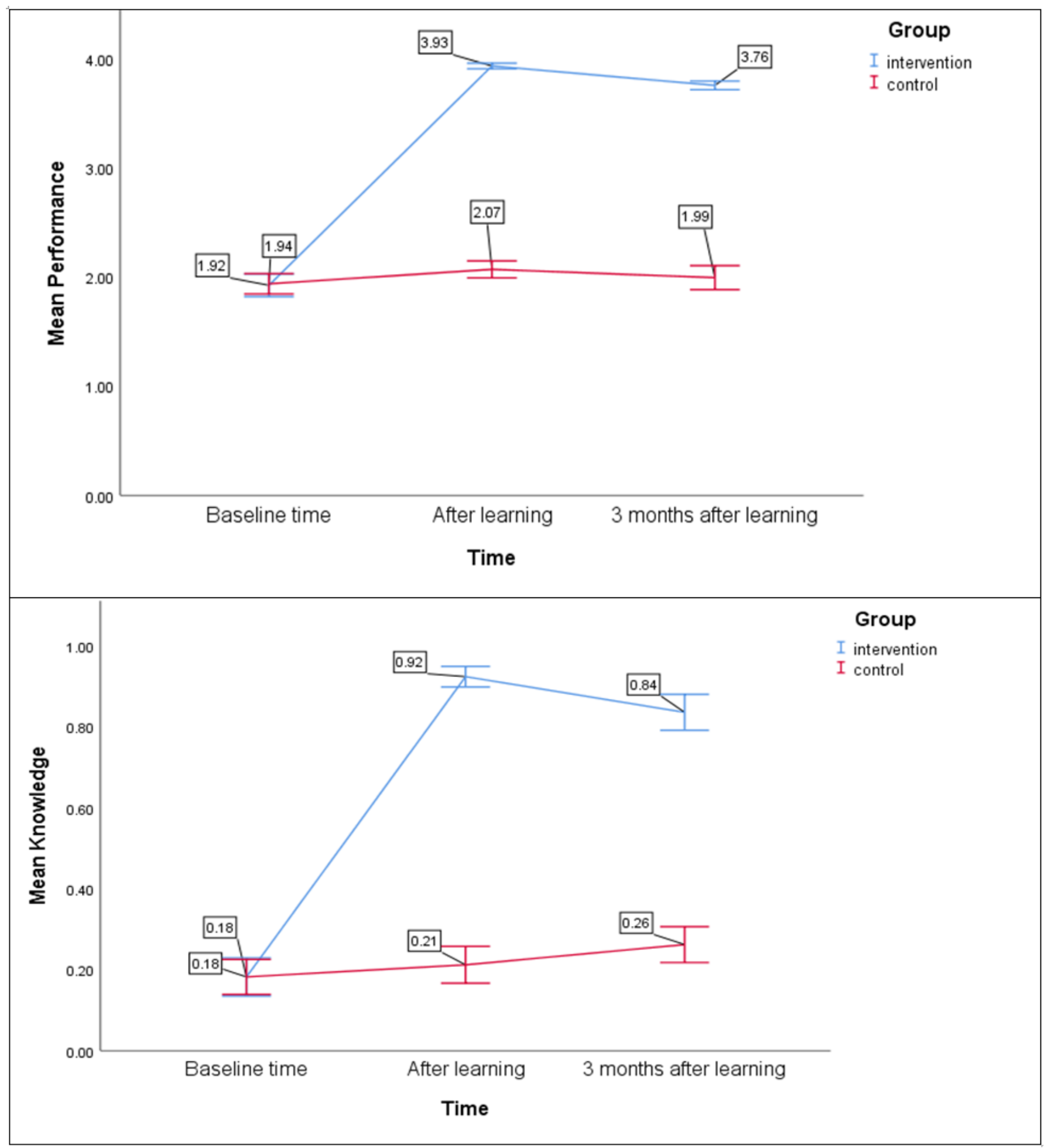

\section{Figure 1}

Error bars to assess the intragroup trends 